\title{
Gait Speed Measurement for Elderly Patients with Risk of Frailty
}

\author{
Xavier Ferre, ${ }^{1,2}$ Elena Villalba-Mora, ${ }^{1}$ Maria-Angeles Caballero-Mora, ${ }^{3}$ Alberto Sanchez, ${ }^{1}$ \\ Williams Aguilera, ${ }^{1}$ Nuria Garcia-Grossocordon, ${ }^{3}$ Laura Nuñez-Jimenez, ${ }^{3}$ \\ Leocadio Rodríguez-Mañas, ${ }^{3}$ Qin Liu, ${ }^{2}$ and Francisco del Pozo-Guerrero ${ }^{1,4}$ \\ ${ }^{1}$ Center for Biomedical Technology, Universidad Politécnica de Madrid, Madrid, Spain \\ ${ }^{2}$ School of Software Engineering, Tongji University, Shanghai, China \\ ${ }^{3}$ Servicio de Geriatría, Hospital Universitario de Getafe, Getafe, Madrid, Spain \\ ${ }^{4}$ Biomedical Research Networking Center in Bioengineering Biomaterials and Nanomedicine (CIBER-BBN), Madrid, Spain
}

Correspondence should be addressed to Xavier Ferre; xavier.ferre@ctb.upm.es

Received 30 June 2017; Revised 22 October 2017; Accepted 29 October 2017; Published 31 December 2017

Academic Editor: Pino Caballero-Gil

Copyright (C) 2017 Xavier Ferre et al. This is an open access article distributed under the Creative Commons Attribution License, which permits unrestricted use, distribution, and reproduction in any medium, provided the original work is properly cited.

The ageing of the population poses a threat to both public and private health and social systems. In the last 50 years, life expectancy has increased by an average of 20 years, and by the year 2050, life expectancy will exceed 90 years of age. However, quality of life in the last years of life is not guaranteed due to conditions such as functional decline and frailty, ultimately progressing to disability. Thus, the detection of such a condition in time is of utmost importance. This paper presents an ultrasonic sensor-based gait speed measurement device controlled via a mobile interface, which permits patients to self-assess physical performance. The system was developed and validated in an iterative process involving a total of 28 subjects ( 21 in the first round and 7 in the second one). After first evaluation at Hospital Universitario de Getafe, some technical problems arose whereas usability testing was well evaluated. The second version addressing the identified issues was technically validated at university premises with good and promising results. Future work envisages deployment of the system developed at subjects' homes to be remotely and unobtrusively monitored.

\section{Introduction}

According to the WHO (World Health Organization), in the last 50 years, life expectancy has increased by an average of 20 years, and if it continues increasing, by the year 2050, life expectancy will surpass 90 years of age [1]. Thus, elderly associated conditions and illnesses are more common today than they were in the past. For these reasons, major efforts are being undertaken investigating and analyzing the effects of aging, in conditions such as frailty, their impact on the cognitive and physical functions, and how to prevent these to improve the quality and duration of life. Frailty is defined as a state of increased vulnerability to adverse outcomes due to a reduction in the ability to respond to stressors [2]. The trajectory of frailty is usually characterized by a progression of functional decline that culminates in dependency and disability $[2,3]$.

The early detection of impeding disease is complex, and a clear algorithm and clinical-friendly screening tools for detection of frailty and/or disability are lacking. Living longer does not necessarily equate to healthy living. Development of early detection tools will permit intervention to prevent or delay the onset of frailty, thus preventing further disability.

Small changes in various health-related metrics could indicate the existence of an underlying condition in the patient. Such a condition might not present with visible symptoms until much later, when it is too late to take preventive measures and very costly to intervene, resulting in lengthy hospital stays and a higher risk for permanent institutionalization. Metrics such as the gait speed are used to measure a person's frailty level [4-6].

Gait speed deterioration is one of the most important indicators of decay in functional ability, with the risk of developing frailty, and later disability [7]. Early detection of any functional decay is critical to improve the quality of life of elderly people and reduce their level of dependency.

Gait speed is typically measured by health professionals in hospital or daycare settings, by manually measuring the time 
the patient takes to walk between two stripes situated in the floor at different distances, depending on the tool used. In the case of the classical SPPB (short physical performance battery) test [8], the distance is 4 meters, but for Fried's criteria, this distance is 4.3 meters [2]. Healthcare professionals use a manual chronometer, which has a risk of inaccuracy and discrepancies between different professionals and different sets of measurements (inter- and intraobserver variability). Additionally, patients may spend several months without getting their gait speed measured, possibly worsening their functional condition to an irreversible state before any health professional can intervene.

This paper presents the design, construction, and validation of an ultrasonic sensor-based gait speed measurement device controlled via a mobile interface, which aims to empower patients in the unsupervised self-assessment of physical performance in both home and hospital settings and to make the measurement more reliable for health professionals.

Next section introduces related work in the subject. Section 3 presents the first version of the proposed device and its clinical evaluation in a hospital environment. Section 4 presents the modified version of the device and the technical validation of this second version. Finally, section 5 presents the conclusions gathered.

\section{Related Work}

Research on systems for gathering health-related metrics, and for measuring gait speed, can be divided into wearableand non-wearable-based devices and sensor networks.

2.1. Wearable Devices. Most of the works related to monitoring and sensorization of medical analysis for elderly patients are based on the usage of wearable devices which gather the data and sometimes even perform simple measures. For example, Tirosh et al. proposed a sensorized sock equipped with pressure sensors to measure the variables for the gait test [9]. Adelsberger et al. proposed equipping a patient with inertial sensors to obtain measures for the gait analysis as well and send these data wirelessly via Bluetooth to a laptop computer for further analysis [10]. In the same line, there are many proposals that make use of different wearable sensors for data collection, such as the one from Morris and Paradiso [11] that advocates integrating the sensors to the patient's insoles and the one from Atallah et al. [12] that proposes to measure variables through a series of accelerometers placed on the patient's ear, among others.

Alternatively, Miura et al. proposed to use a mobile phone hung around the neck and fitted inside the user's jacket to measure walking characteristics, such as walking distance, time taken, speed, balance functions, body sways, and the number of steps taken. However, its accuracy for gait speed is too low for the SPPB test [13].

Finally, ultrasound sensors have been used to estimate the gait speed of subjects embedded with wearable devices; Weir and Childress proposed a device that combines ultrasound pulses and infrared technology to determine the real-time position of a user with respect to a base unit. Gait speed profile analysis provides parameters such as the cadence, step length, or step time variation [14].

The main shortcoming of the usage of these wearable devices that need to be attached to the patient in some way, is their intrusiveness. They may alter the conditions in which the analysis is performed, affecting users' behavior, and in some cases, they could be incompatible with some users' conditions. For example, some patients use orthopedic insoles on a daily basis, which makes it impossible to introduce a second insole to measure walking variables. The same issue can occur with socks or shoe-coupled devices as well as other nonwearable devices.

Some proposals use diverse ambient sensors for gathering vital variables. One of the most-used devices for this purpose is the Kinect ${ }^{\circledast}$, developed by Microsoft, which groups a series of cameras and infrared sensors, allowing it to detect depth, record videos, and analyze movements and posture, among other features. Several research papers are based on this device, such as $[15,16]$, taking advantage of its resources to record the measures and then to model a series of significant parameters, such as longitude of the stride, walking speed, and walking direction, among other measures. These proposals are designed to work in the patient's home and collect all possible data for further analysis, obtaining extrainformation through modeling that could be important for the health professional. Kinect-based systems may raise privacy concerns, since it is composed of a camera recording users.

Other studies, like [17, 18], propose sensorized mats, based on a pressure sensor matrix, for obtaining these variables. These solutions are mostly restricted to use inside a health facility, operated by health professionals in a controlled environment. They are too costly and too difficult to calibrate for use in a home setting.

When considering in-house monitoring systems, there is an added difficulty in the analysis and evaluation of data because the measured events occur without monitoring, as opposed to the controlled environment of a medical office. When the patient is at home, there is no easy way of detecting when a walk begins and when it ends, if there are external factors affecting the speed and direction of the walk (e.g., if the patient is carrying something) or if there is some other variable affecting the measurement. Stone and Skubic minimize the "noise" introduced by these factors through the application of decision models, algorithms, and the gait variables to identify "valid" walks [16]. Van Den Broeck et al. proposed in their investigation a formula, using global coherence field (GCF), to estimate and remove the possible effects of the aforementioned noise [19].

2.2. Sensor Networks. Some research efforts have been focusing on behavioral monitoring, such as how to use sensor networks to help in the early detection of medical problems and to monitor adherence to doctors' recommendations regarding lifestyle and medication intake for chronic illness [20].

The University of Rochester built a smart medical home in their Center for Future Health, filled with different types of sensors, functioning as a laboratory, to test and develop various gadgets, sensors, and applications, that could be used as part of a health-integrated system [21]. Similarly, the Aware 
Home in Georgia Tech, is a 3-storey, 5040 square feet facility designed to facilitate research in three main areas: health, entertainment, and sustainability, investigating how new technologies can impact the lives of people at home [22].

Implementing home sensors could give the elderly population the perfect balance between independence and proper monitoring. For example, Zouba et al. proposed the implementation of multiple sensors attached to furniture at home, combined with cameras for video analysis to monitor the behavior of elderly people performing everyday activities, such as cooking meals and eating [23]. Wood et al. took behavioral monitoring a step further by combining it with assisted living. They took data gathered from multiple heterogeneous sensors and fed them into a backend layer that analyzes and combines the data in order to produce decisions that could influence other devices, such as a power manager [24]. This system was thought to be extensible with the ability to add more sensors and user interfaces, which is a similar objective to the one proposed in this work. As with many other proposed solutions, it uses a proprietary query software language called SensQ, developed solely for this system which makes it harder for other consumers to adopt it. It also has a closed architecture which makes it complicated to integrate 3rd party external developments. These two limitations usually result in increased deployment costs and the risk of obsolescence.

\section{UltraGaitSpeed System v1.0}

For the measurement of the gait speed in a home setting, we have designed a lightweight and low-cost ultrasonic sensorbased system called UltraGaitSpeed. The sensor device connects via Bluetooth with an Android mobile phone that logs the measured data and allows the user to control the system. When an Internet connection is available, the data are sent to a Cloud server.

Our approach to gait speed measurement aims to offer a lightweight and unobtrusive solution that liberates the user from wearing any kind of gadgets and takes advantage of a very powerful device (modern smartphones) that has a tremendous processing power and good quality/price ratio.

A strip of light sensors with an Arduino board can be easily deployed in a house and can provide the necessary information to monitor decay in gait speed, possibly indicating more serious health problems that require immediate assistance. The gathered information is uploaded to a server and securely connected to the HIS (hospital information system), so physicians can monitor patient evolution, and alarms can be raised in a clinical setting when the condition of a patient rapidly deteriorates.

3.1. System Design. We chose HC-SR04 ultrasonic sensors for their lightweight and low-cost characteristics. This sensor uses sonar, sending a signal from one side and receiving it on the other, to measure the distance of an object in front of it by subtracting the time between both signals. This means that we can detect when an object moves in front of the sensor by tracking the distance variations between measures. Since these sensors emit an ultrasonic sound wave and sound waves dissipate in a cone-shaped way, we need to perform some tests with the sensors to make a decision about the number of sensors and to establish a minimum distance between sensors to avoid interference (Figure 1).

After extensive testing, we determined that 35 centimeters is the minimum distance between sensors for them to work without interference and that 5 sensors provided enough accuracy since additional sensors did not improve the measurements (Figure 2).

The 5 sensors are connected to an Arduino UNO board and set up with 1-meter separation between them along a flexible EVA foam strip. The resulting 4-meter strip can serve to reproduce the SPPB gait speed test used by health professionals.

In order to cover the sensors and protect the cables, cases were printed in 3D for every sensor and its connections (Figure 3). This provides the required robustness for a solution to be installed in a home setting.

The device runs a simple software that loops through the connected sensors, sends a signal, and listens for the response, which is stored in a local variable. After looping through all the sensors, it sends via Bluetooth the data gathered from the sensors in JSON format, with the number of milliseconds relative to the system start as a timestamp.

The mobile application allows the user to calibrate the sensors, start the measurement, and get data results on the screen. It also stores measurements in an internal log, which is uploaded to a server when an Internet connection is available.

This first version of the UltraGaitSpeed system works according to the flowchart shown in Figure 4. The application guides the user in all the steps required for system operation, namely, Bluetooth enabling, sensor calibration, step measurement, and final result display.

Bluetooth connection is enabled in two steps. First, the application checks the activation of Bluetooth in the device and then connects with the Arduino Board. Next, the sensor calibration starts, using a series of iterations to make sure that stable distances are measured by each sensor. These distances are the baseline for the later measurements. If there is movement in front of any sensor, the calibration will fail. If there is a failure in any of these steps, the application displays an error message to the user.

When calibration finishes, the system starts measuring gait speed. Measurement is achieved by means of detection events. When a user is detected by a sensor, the distance measured deviates from the sensor baseline measure and a timestamp is logged. Finally, when every sensor has detected the patient, the passing time at each sensor and the overall time are calculated by subtraction.

By pressing the Restart button on the screen, the process can start again for a new measurement.

3.2. Evaluation. Even if our system is meant to be used by patients and informal caregivers in an extrahospital setting, we need to validate that the system gathers gait speed measurements as reliably as the manual measurement by health professionals in medical facilities. 


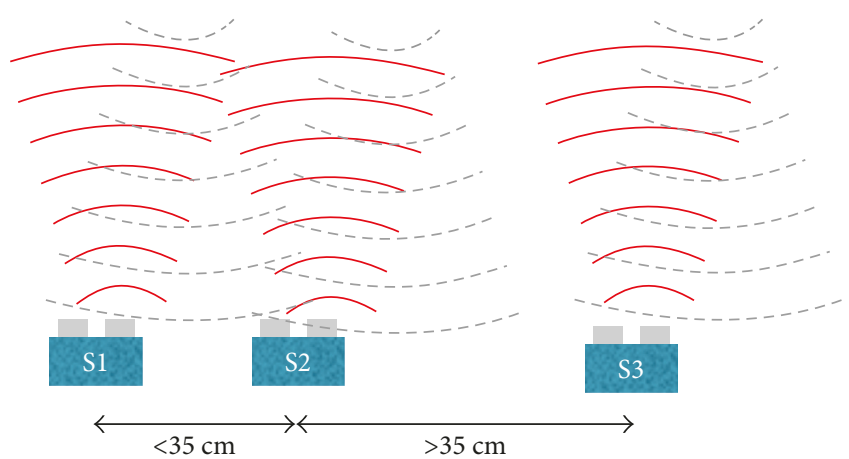

Figure 1: Minimum distance between sensors.

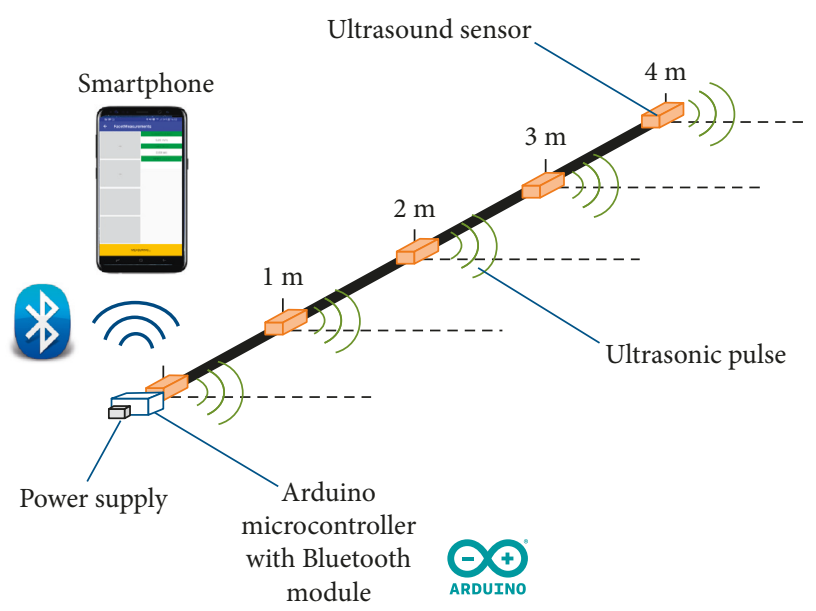

FIGURE 2: Equidistant sensor placement in the strip.

For that purpose, we carried out an experiment with older patients attending the Day Hospital in the Geriatric Service at the Hospital Universitario de Getafe (HUG). The objective is to validate the accuracy and feasibility of the proposed measurement solution as compared to the current manual practice.

3.2.1. Experiment Design. Our hypothesis is that the usage of the proposed ultrasound sensor-based solution allows the conduction, in a precise way, of the SPPB gait analysis, with a difference threshold of \pm 0.5 seconds compared to the manual measures, which is the current practice in Geriatric Service at HUG.

The inclusion criteria for the patients to take part in the experiment were as follows:

(i) Older than 70 years

(ii) Patient of the Geriatric Service at HUG.

The exclusion criteria were as follows:

(i) Cognitive impairment: minimental test result lower than 20

(ii) High degree of disability: Barthel index lower than 60 .

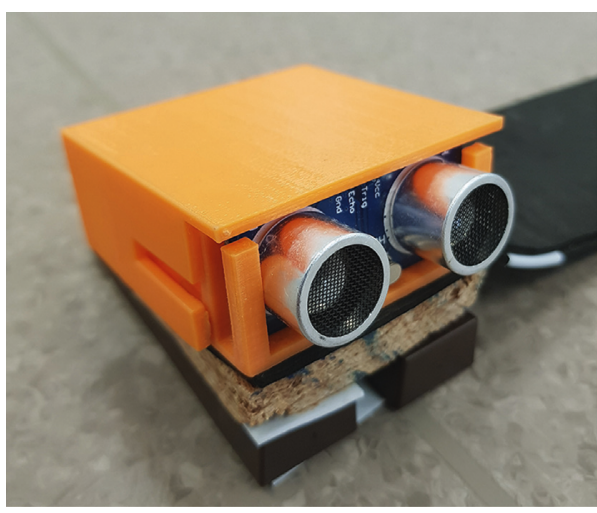

FIGURE 3: 3D printed case for covering sensors and connections.

The patients were randomly selected from those that come to the Day Hospital, with at least 2 patients selected in each one of the following groups:

(i) Patients that do not need any technical help for walking

(ii) Patients that need a cane for walking

(iii) Patients that need a walker for walking.

Two separate tests were conducted with each patient. First, the gait test as described in point 2 of the SPPB analysis was used to measure the speed of the walk, having the patient standing right on the start line of the 4-meter strip, including acceleration speed (static start). Second, an ordinary gait test was performed, having the patient start a meter before the starting line (dynamic start) in order to exclude acceleration from the measure.

The experiment was designed to simultaneously measure using both the current manual method and the proposed system. The manual method is done with the patient walking between two green lines while the health professionals measure with a stopwatch. For each of the tests, three measurements were taken in total: two separate manual measures and one with the proposed solution, all of them done by health professionals experienced in measuring gait speed. None of the health professionals involved in taking the measures could see the measures taken by the others.

Forms for the manual measure results were prepared as well as an SUS (System Usability Scale) [25] satisfaction questionnaire and an impressions questionnaire to gather information about the system usability from the health professional using our proposed system in the experiment.

The current protocol for gait speed measurement for the SPPB test was followed: measuring two walks for each patient and taking as valid the best of both measures.

The Ethical Committee of the University Hospital of Getafe approved the protocol.

3.2.2. Experiment Execution. On the day of the experiment, we set up the data gathering device and the mobile application to conduct the tests. The setting up of the gathering device was a very simple procedure, taking less than 5 minutes. 


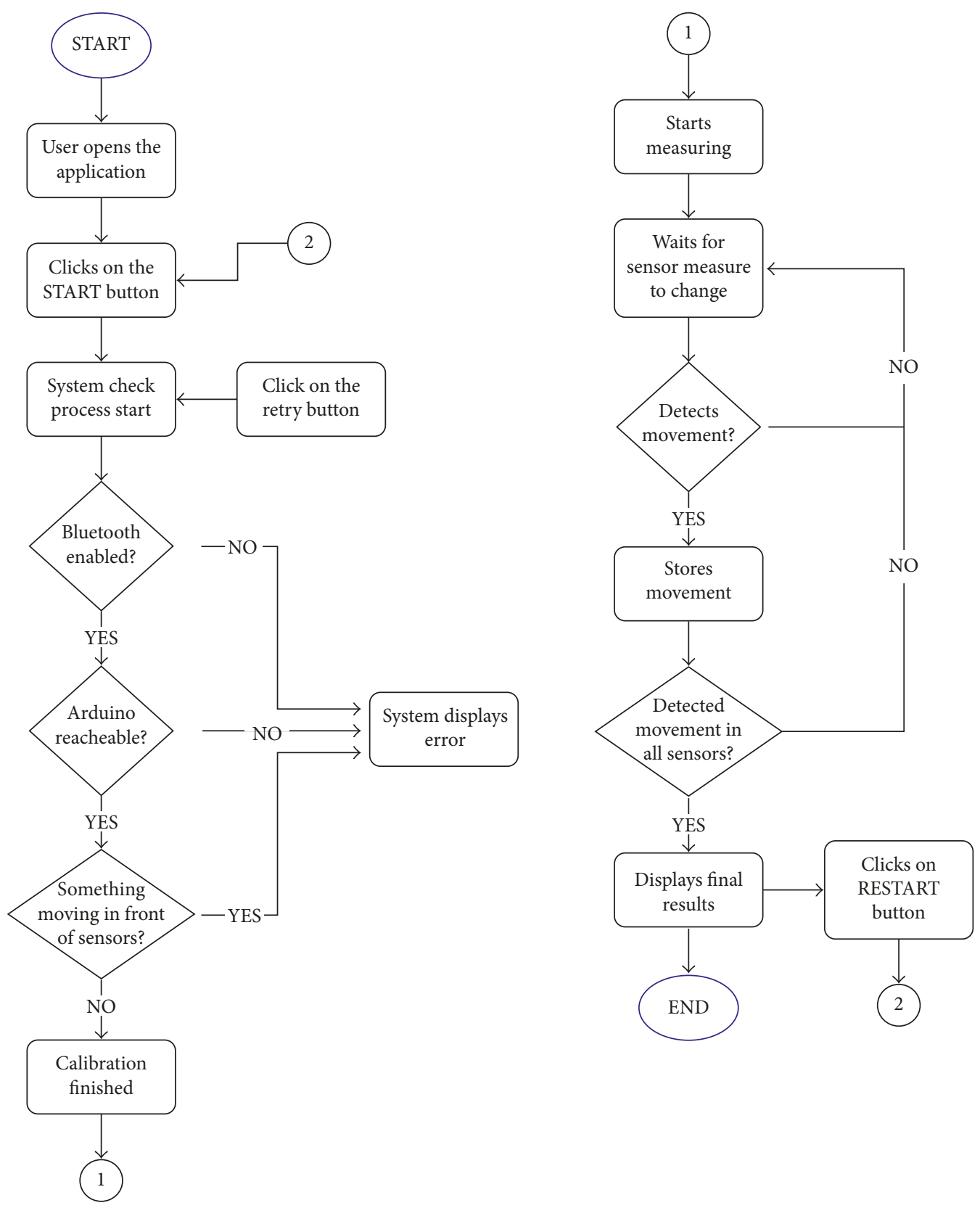

FIgUre 4: Algorithm for UltraGaitSpeed v1.0.

Three health professionals volunteered to participate in the experiment as data gatherers, among whom we randomly distributed the roles for manual collection and usage of the application. After selecting the professionals, we gave them a brief explanation of the experiment and their tasks and provided them with the individual data collection forms. A mobile device that had the application already installed was provided to the health professional for taking measurements.

The experiment was conducted as follows:

(1) After selecting those patients that met the inclusion criteria and that expressed willingness to participate (by signing the informed consent), the health professional proceeded to explain what the test consisted of.
(2) The subjects were asked to carry out the two tests (the static and dynamic start). Their gait speed was measured by health professionals. One professional measured gait speed automatically with the proposed system, and the two other professionals performed the test manually using the current method (stopwatch). Manual results were collected in an ad hoc paper form.

(3) After finishing with all the patients of the study, we gathered the paper forms from the health professionals and asked the one that was using the application to complete the satisfaction and impressions questionnaires. 
After carrying out the experiment, an analysis of the data was conducted to ensure the feasibility of the system through the analysis of the following variables:

(i) Number of measures that ended correctly

(ii) Number of measures that had to be repeated

(iii) Time for the preparation of each test

(iv) Deviation related to the control measure.

The experiment was conducted with 21 voluntary patients that complied with the inclusion and exclusion criteria. Their average age was 81 years, with the youngest patient being 71 and the oldest 90 . The number of patients with technical aids, such as canes and walkers, was 7 from the total of 21 patients that were included in the study.

The experiment with patients went smoothly with no need for further intervention from the researchers. There were slight problems with the calibration in patients who use walking canes, which were resolved after rebooting the system.

3.2.3. Results. After finishing the experiment, we proceeded with the extraction of data gathered from the forms and the questionnaires completed by the health professional using the application.

First, we merged the data gathered into one single spreadsheet, grouped by a type of measure (stopwatch 1 and 2 and application). After this, a series of data treatments were applied to the data as follows:

(1) We selected as final measure for each subject the lower measure between the two that were taken for each one of the tests (the dynamic and static start), as is usually done by health professionals when performing this test.

(2) With the final measure for each of the subjects, we calculated the average between the manual measures taken with the stopwatch to compare those measures with the ones obtained from the application.

(3) We then proceeded to calculate the difference module of the measures obtained with the manual procedure, and those from the application, and all the combinations between them:

(a) Between manual measure 1 and manual measure 2

(b) Between the average of the manual measures and the application

(c) Between manual measure 1 and the application

(d) Between manual measure 2 and the application.

(4) Having these measures, we proceeded to calculate statistical variables of the differences. These are relevant in order to measure the degree to which the application was able to get correct measures in the experiment. The variables obtained were the mean, the median, the mode, and the truncated mean.

(5) We decided to calculate the truncated mean of the differences with a threshold of $10 \%$. This allowed us to remove $10 \%$ of outliers from the calculation of the mean (which accounted for 2 measures), thus omitting occasional errors that the application might have had.

After data processing, we obtained the results detailed in Table 1 for the case of the measure with no acceleration and in Table 2 for the case of the measure including the patient's acceleration. Overall relevant variables of the experiment are shown in Table 3.

Regarding the satisfaction questionnaire, the responses produced an SUS score of 72.5, which is slightly over the average of usability scores taken as a reference for an SUS score of 68.5. This shows that there is satisfaction in the user but not to an exceptional level.

The open impressions questionnaire shows a favorable perception from the health professional, and it offers several relevant suggestions for improvement:

(i) To optimize calibration and to not require the patient to wait, standing without movement while the system calibrates. Elderly patients have difficulty standing for some time, since they get easily tired. In some cases, there is a risk that they lose their balance

(ii) To avoid having to perform a calibration every time, a measure is started. The system could calibrate only the first time, providing a button to recalibrate when the health professional sees fit

(iii) To extract additional variables from the sensors, such as direction of the walk, variability of the speed, stride length, step length, and others. For the implementation of this suggestion, we will need to review and perform a deeper analysis of the gathered data, in order to determine which of these proposed new variables can be obtained from the current sensors.

3.2.4. Discussion. These results show the feasibility of deploying our proposed system in a clinical setting and having health professionals use it. But the reliability of the measures falls below the threshold established of a maximum difference of 0.50 seconds between the manual measure and the automatic measure. Considering that all the measures fall in the interval 2.15-9.47 seconds, average differences of 0.64 or 0.67 are too high.

Further detailed study of the results with health professionals at HUG unveiled that the algorithm used in UltraGaitSpeed v1.0 for measuring gait speed did not exactly comply with the measuring criteria used in classical gait speed tests, as routinely performed at HUG. Both for the static and dynamic start gait speed tests, the event triggering the start and finish of measuring is the moment when the last heel of the subject reaches the mark on the floor, while the algorithm implemented in v1.0 of our solution starts measuring when the sensor detects the first foot. This is why the presence of walking aids produced some errors.

Regarding calibration, the system failed several times for the static start gait speed tests. In this case, the subject stands right in front on the starting line, with the tip of his foot on it, and waits for the command to go. Even the smallest variation in the distance detected at the starting line, due to subjects' 
TABLE 1: Experiment results for the dynamic start measure with UltraGaitSpeed v1.0.

\begin{tabular}{lcccc}
\hline & & \multicolumn{2}{c}{ Differences (seconds) } \\
& $\begin{array}{c}\text { Stopwatch 1 and } \\
\text { stopwatch 2 }\end{array}$ & $\begin{array}{c}\text { Stopwatch avg. and } \\
\text { UltraGaitSpeed v1.0 }\end{array}$ & $\begin{array}{c}\text { Stopwatch 1 and } \\
\text { UltraGaitSpeed v1.0 }\end{array}$ & $\begin{array}{c}\text { Stopwatch 2 and } \\
\text { UltraGaitSpeed v1.0 }\end{array}$ \\
\hline Mean & 0.13 & 0.66 & 0.66 & 0.67 \\
Median & 0.06 & 0.71 & 0.70 & 0.63 \\
Mode & 0.03 & No mode present & 0.75 & 1.13 \\
Truncated mean (10\%) & 0.10 & 0.62 & 0.62 & 0.62 \\
Average relative error with respect & $2.8 \%$ & $13.6 \%$ & $13.7 \%$ & $14.0 \%$ \\
to manual watch average time (\%) & 0.13 & 0.45 & 0.42 & 0.50 \\
Standard deviation & & & \\
\hline
\end{tabular}

TABLE 2: Experiment results for the static start measure with UltraGaitSpeed v1.0.

\begin{tabular}{lcccc}
\hline & & \multicolumn{2}{c}{ Differences (seconds) } \\
& $\begin{array}{c}\text { Stopwatch 1 and } \\
\text { stopwatch 2 }\end{array}$ & $\begin{array}{c}\text { Stopwatch avg. and } \\
\text { UltraGaitSpeed v1.0 }\end{array}$ & $\begin{array}{c}\text { Stopwatch 1 and } \\
\text { UltraGaitSpeed v1.0 }\end{array}$ & $\begin{array}{c}\text { Stop watch 2 and } \\
\text { UltraGaitSpeed v1.0 }\end{array}$ \\
\hline Mean & 0.20 & 0.64 & 0.64 & 0.64 \\
Median & 0.13 & 0.45 & 0.38 & 0.49 \\
Mode & 0.28 & No mode present & 0.13 & 0.47 \\
Truncated mean (10\%) & 0.16 & 0.55 & 0.53 & 0.56 \\
Average relative error with respect & $3.7 \%$ & $11.9 \%$ & $0.9 \%$ & $11.9 \%$ \\
to manual watch average time (\%) & 0.20 & 0.57 & 0.63 & 0.53 \\
Standard deviation & & &
\end{tabular}

or walking aids' movement, triggered the timing and produced irregular measurements.

Additionally, the system requires that the test is carried out only in one direction, while software could detect the walking direction and thus capture two measurements per patient without requiring him or her to walk back to the starting point. The patient could just turn around after the first measurement and walk in the other direction for the second measure.

\section{UltraGaitSpeed v2.0}

In order to overcome the main problems identified for v1.0, we designed a new algorithm (Figure 5) and a new version for the mobile app (Figure 6), the UltraGaitSpeed v2.0.

The new algorithm considers the last detection event in a sensor to be the moment to consider for user passing such a sensor (Figure 7). This better fits the moment when the patient's heel leaves the sensor detection field. This moment is used for both establishing the start and finish of the measure, mirroring the way the health professionals define the start and finish time when performing this test. Likewise, this criterion determines partial timing.

The distance between sensors is fixed and known, and the detection field angle is the same for all of them. Therefore, if we assume that the patient will walk straight, the point where the last foot abandons each detection field will be separated by the same distance as the sensors themselves.

So, with v2.0 of our device, we can measure the time spent moving from sensor A to sensor B.
TABLE 3: Variables obtained from the experiment with UltraGaitSpeed v1.0.

\begin{tabular}{lc}
\hline Variable & Result \\
\hline $\begin{array}{l}\text { Time for the preparation } \\
\text { of each test }\end{array}$ & Mean of 4 minutes \\
$\begin{array}{l}\text { Deviation related to the } \\
\text { control measure }\end{array}$ & Mean of 0.58 seconds \\
Maximum time measured & 9.47 seconds \\
Minimum time measured & 2.15 seconds \\
\hline
\end{tabular}

Figure 5 depicts the new algorithm designed for v2.0 of our system. When one of the sensors detects the user, it is activated and waits for the field-of-view exit event, at which point the timestamp is registered. In order to make sure that the exit event was actually the last part of the body to pass (and not, e.g., just the first foot or a walking cane), the measurement for each segment of the gait speed test is not confirmed until the exit event is produced in the next sensor in the strip. (The last sensor has a timeout for confirmation, since it has no other sensor next to it.) In the case of a new detection event before confirmation in the following sensor, the sensor waits for the expected exit event and then the timestamp is reset.

This new algorithm entails the following advantages:

(1) It replicates the measuring criteria used in the classical gait speed tests carried out by professionals in clinical settings. 


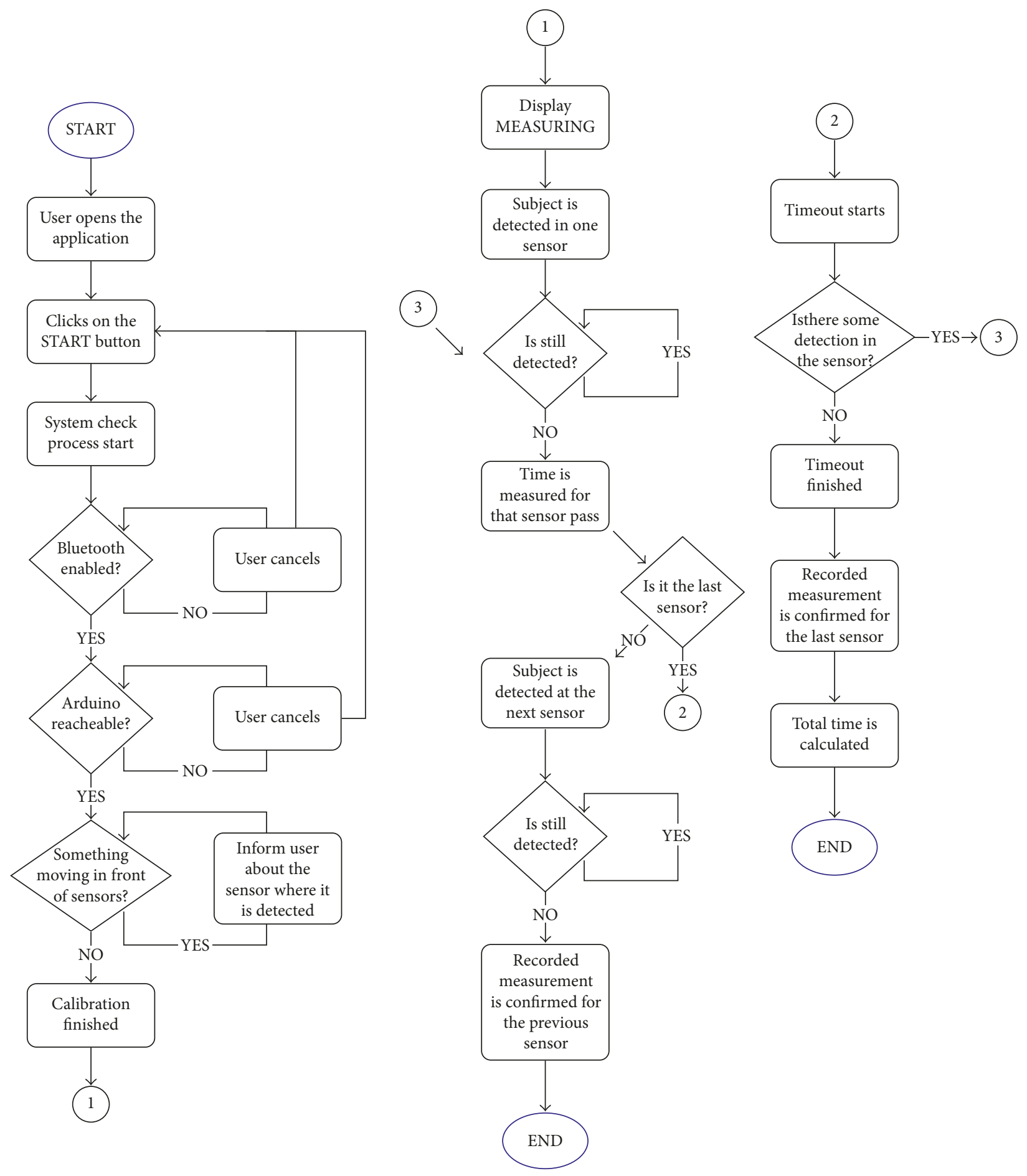

FIGURE 5: Algorithm for UltraGaitSpeed v2.0.

(2) Start time is automatically triggered when the patient leaves the first sensor, instead of requiring an action in the mobile app.

(3) Calibration does not require that the patient waits motionless just before the first sensor. It is carried out with the patient positioned behind or far away from the sensor strip, and the measuring will automatically start when the patient goes through the first sensor. We will thus avoid errors arising from the small distance variations while patients wait for the calibration to end.

4.1. Evaluation. We have evaluated this new version of our proposal with seven users between 68 and 79 years of age, six of them with mobility problems and two requiring a cane. 


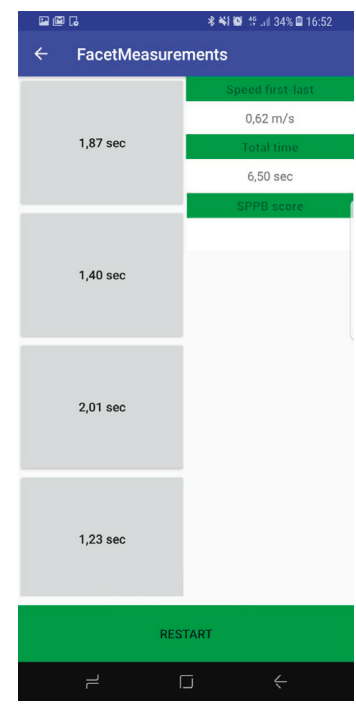

FIgURE 6: Screen capture of the mobile app for UltraGaitSpeed v2.0.

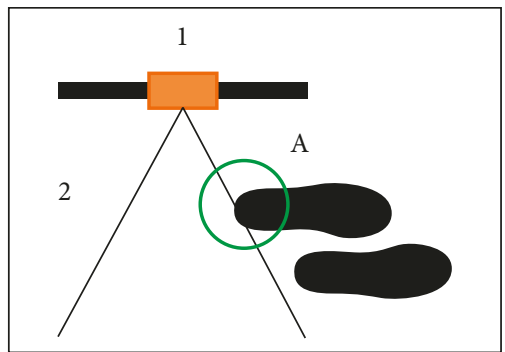

FIGURE 7: Detection moment for the user passing through a sensor.

We have deployed UltraGaitSpeed v2.0 at CTB with indications of the place for subjects to position themselves for both the dynamic and the static start tests. We have briefly explained how the system works, and how it is controlled from the mobile app to the subject, in the same way as we foresee system installers doing when the system is actually used in a home setting.

Each participant is asked to measure two walks with a static start and two more with a dynamic start, in total providing four measures to the system.

A biomedical engineer trained at HUG on the SPPB test measures manually with a stopwatch the time spent by the user between the two extremes of the sensor strip (4 meters). The measurer followed the criteria used in regular clinical practice. Timing must be recorded when the last heel of the patient passes over the line that marks the start and finish of the four-meter walk.

4.1.1. Results. The results obtained for timing were analyzed replicating the protocol of UltraGaitSpeed v1.0 evaluation but having only one stopwatch measurement as a reference for comparison.

The results obtained are presented in Tables 4 and 5 .
TABLE 4: Experiment results for the static start measure with UltraGaitSpeed v2.0.

\begin{tabular}{lc}
\hline Differences (seconds) & \\
UltraGaitSpeed v2.0 and stopwatch & 0.35 \\
\hline Mean & 0.29 \\
Median & None \\
Mode & 0.35 \\
Truncated mean (10\%) & $7.39 \%$ \\
Average relative error with respect to manual watch & \\
mean time (\%) & 0.22 \\
\hline
\end{tabular}

TABle 5: Experiment results for the dynamic start measure with UltraGaitSpeed v2.0.

\begin{tabular}{lc}
\hline Differences (seconds) & \\
UltraGaitSpeed v2.0 and stopwatch & 0.15 \\
\hline Mean & 0.19 \\
Median & None \\
Mode & 0.15 \\
Truncated mean (10\%) & $4.13 \%$ \\
Average relative error with respect to manual watch & \\
mean time (\%) & 0.10
\end{tabular}

The new algorithm demonstrated a greater reliability for measurement than the one implemented in the previous version of the device. Both the absolute and relative errors between the stopwatch and UltraGaitSpeed v2.0 have been reduced. In fact, the average absolute error is smaller than the established accepted error of 0.5 seconds. Moreover, the standard deviation of the error is smaller compared with the first version, which suggests the error of this version falls in a smaller range, and therefore is more predictable.

The timing problem associated with the use of walking aids has been solved in this new version. The trials with two patients who use walking aids demonstrated the reliability of UltraGaitSpeed v2.0. The average relative error for their tests was smaller than the overall $(3.95 \%)$, and the measurer did not report any incident related to this issue.

Observation of subjects while using the system and open conversation with them have provided insight about some usability problems that need to be addressed as follows:

(i) The word "measuring..." conveys the idea that the time watch is already ticking, while it actually means that the sensors have been calibrated and are already working to detect whatever passes by.

(ii) Error and warning messages, such as the one appearing when the timeout for the last sensor goes off, need to be reconsidered to speak the user's language, and some of them should not even be shown unless the app is in developer mode.

Despite the room for improvement, UltraGaitSpeed v2.0 was accepted by the users. They completed the 


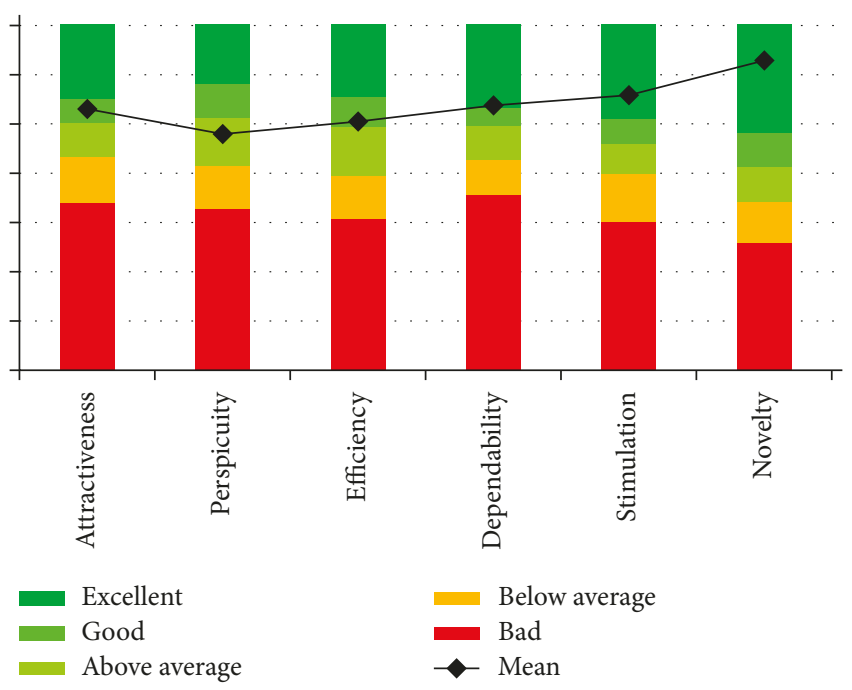

FIgURE 8: Results for UEQ.

System Usability Scale (SUS) and User Experience Questionnaire (UEQ) [26] after performing the gait speed tests, giving an average SUS score for UltraGaitSpeed v2.0 of 73.5 .

Studies have demonstrated that the average SUS score is 68 . Therefore, we have shown that our system is more usable than the benchmark and is in the second quartile [27].

In the case of the UEQ, we obtained excellent results in Novelty and Stimulation, good results in Attractiveness, Efficiency, and Dependability, and results above the average in Perspicuity (Figure 8).

4.1.2. Discussion. Despite the small size of the study population, results suggest that UltraGaitSpeed v2.0 may be used in unsupervised environments for evaluating the gait speed of patients, from a technical point of view.

Additionally, the new algorithm prevents one of the more recurrent errors of UltraGaitSpeed v1.0. The initial algorithm triggered incorrect start of timing when small movements of patients waiting to begin the static start test were detected as actual movement.

With these results, we confirm that the new design eliminates the error in the start of each measure, by having the measuring algorithm consider the moment when the user leaves each field of detection as the time of passing.

The validation of UltraGaitSpeed v2.0 has shown the system feasibility in its usage by the intended audience: elderly people. Participants in the validation have shown a favorable opinion of the usefulness and usability of the system, even though they suggested necessary improvements.

Further validation is necessary in the intended final environment: patients' homes. A longitudinal study is necessary to better understand how the system can be regularly used by its intended users.

\section{Conclusions}

We have presented two versions of UltraGaitSpeed (v1.0 and v2.0), a sensor-based system controlled through a mobile app for measuring gait speed, aimed to be used by patients themselves or their caregivers.

Through the two-cycle validation and redesign process, the proposed system has shown its usefulness to automatically measure gait speed through a low-cost and lightweight solution that can be easily deployed in any medical facility, in a daycare center, or even in a private home. As an Android app controls the system, this allows its usage in the patient's or informal caregiver's own mobile phone, thus further reducing the cost of the integrated solution.

Even if the validation has been carried out in a controlled environment with health professionals or researchers, the proposed system can be part of an ambient-assisted living network of sensors, that contributes to a better quality of life and earlier detection of deteriorating conditions that require professional medical help.

The usability level of the system needs to be improved with a new redesign-evaluation loop, where acceptability and usability issues are resolved.

The next step in our research is to validate the system in a hospital setting like the one used in the validation of the first version and a longitudinal study carried out in inhome settings. In these studies, we will inquire about the most appropriate amount of sensors and length of the strip. The aim is to reduce the strip to a length of 2 meters, facilitating its reliable usage in home environments with limited space.

The system will be extended to address other SPPB test parts and other elements from the comprehensive geriatric assessment like the chair stand test (measuring how long the patient takes to sit down in a chair and stand up again five times), involuntary loss of weight, and the items of the Linda Fried criteria of frailty. We will provide these elements either through sensor-based systems like the one presented in this paper or through an extension of the mobile app.

At a subsequent stage, the aim is to create a system based on UltraGaitSpeed that measures gait speed unobtrusively, without the need for the user to actively start the measuring. If the sensor strip can be inconspicuously installed in a corridor wall and measure gait speed every time the user passes by, it will provide much richer information without the added stress of following instructions and manipulating a mobile phone.

\section{Conflicts of Interest}

The authors declare that there are no conflicts of interest regarding the publication of this paper.

\section{Acknowledgments}

This research was funded by the EIT Health in the FACET (FrAilty Care and wEll funcTion) project and by funds from CIBERfes (FEDER). The authors would like to thank all the 
volunteers that helped them to validate their solution acting as test subjects and to thank the reviewers for their insightful comments.

\section{References}

[1] D. M. Ediev, "Life expectancy in developed countries is higher than conventionally estimated. Implications from improved measurement of human longevity," Journal of Population Ageing, vol. 4, no. 1-2, pp. 5-32, 2011.

[2] L. P. Fried, C. M. Tangen, J. Walston et al., "Frailty in older adults: evidence for a phenotype," Journals of Gerontology Series A: Biological Sciences and Medical Sciences, vol. 56, no. 3, pp. M146-M157, 2001.

[3] T. M. Gill, E. A. Gahbauer, L. Han, and H. G. Allore, "Trajectories of disability in the last year of life," New England Journal of Medicine, vol. 362, no. 13, pp. 1173-1180, 2010.

[4] M. Runge and G. Hunter, "Determinants of musculoskeletal frailty and the risk of falls in old age," Journal of Musculoskeletal and Neuronal Interactions, vol. 6, no. 2, pp. 167-173, 2006.

[5] J. M. Hausdorff, D. A. Rios, and H. K. Edelberg, "Gait variability and fall risk in community-living older adults: a 1-year prospective study," Archives of Physical Medicine and Rehabilitation, vol. 82, no. 8, pp. 1050-1056, 2001.

[6] J. M. Hausdorff, A. Schweiger, T. Herman, G. YogevSeligmann, and N. Giladi, "Dual-task decrements in gait: contributing factors among healthy older adults," Journals of Gerontology Series A: Biological Sciences and Medical Sciences, vol. 63, no. 12, pp. 1335-1343, 2008.

[7] E. Villalba Mora, R. Petidier-Torregrossa, C. Alonso-Bouzon, J. A. Carnicero-Carreño, and L. Rodríguez Mañas, "Early detection of Heart Failure exacerbation by telemonitoring in old people," International Journal of Integrated Care, vol. 15, no. 5, pp. 116972-116974, 2015.

[8] J. M. Guralnik, E. M. Simonsick, L. Ferrucci et al., "A short physical performance battery assessing lower extremity function: association with self-reported disability and prediction of mortality and nursing home admission," Journal of Gerontology, vol. 49, no. 2, pp. M85-M94, 1994.

[9] O. Tirosh, R. Begg, E. Passmore, and N. Knopp-Steinberg, "Wearable textile sensor sock for gait analysis," in Proceedings of the 2013 Seventh International Conference on Sensing Technology (ICST), pp. 618-622, IEEE, Wellington, New Zealand, December 2013.

[10] R. Adelsberger, N. Theill, V. Schumacher, B. Arnrich, and G. Tröster, "One IMU is sufficient: a study evaluating effects of dual-tasks on gait in elderly people," in Proceedings of the International Conference on Wireless Mobile Communication and Healthcare, pp. 51-60, Springer, Paris, France, November 2012.

[11] S. J. Morris and J. A. Paradiso, "Shoe-integrated sensor system for wireless gait analysis and real-time feedback," in Proceedings of the Second Joint 24th Annual Conference and the Annual Fall Meeting of the Biomedical Engineering Society EMBS/BMES Conference 2002 (Engineering in Medicine and Biology, 2002), vol. 3, pp. 2468-2469, IEEE, Houston, TX, USA, October 2002.

[12] L. Atallah, O. Aziz, B. Lo, and G. Z. Yang, "Detecting walking gait impairment with an ear-worn sensor," in Sixth International Workshop on Wearable and Implantable Body Sensor Networks, 2009 (BSN 2009), pp. 175-180, IEEE, Berkeley, CA, USA, June 2009.
[13] T. Miura, K. I. Yabu, A. Hiyama, N. Inamura, M. Hirose, and T. Ifukube, "Smartphone-based gait measurement application for exercise and its effects on the lifestyle of senior citizens," in Proceedings of the Human-Computer Interaction (INTERACT 2015), pp. 80-98, Springer, Bamberg, Germany, September 2015.

[14] R. F. Weir and D. S. Childress, "A portable, real-time, clinical gait velocity analysis system," IEEE Transactions on Rehabilitation Engineering, vol. 5, no. 4, pp. 310-321, 1997.

[15] M. Rantz, M. Skubic, C. Abbott, Y. Pak, E. E. Stone, and S. J. Miller, "Automated fall risk assessment and detection in the home: a preliminary investigation," in Proceedings of the AAAI Fall Symposium: Artificial Intelligence for Gerontechnology, Arlington, VA, USA, November 2012.

[16] E. E. Stone and M. Skubic, "Mapping Kinect-based in-home gait speed to TUG time: a methodology to facilitate clinical interpretation," in Proceedings of the 2013 7th International Conference on Pervasive Computing Technologies for Healthcare (PervasiveHealth), pp. 57-64, IEEE, Venice, Italy, May 2013.

[17] A. L. McDonough, M. Batavia, F. C. Chen, S. Kwon, and J. Ziai, "The validity and reliability of the GAITRite system's measurements: a preliminary evaluation," Archives of Physical Medicine and Rehabilitation, vol. 82, no. 3, pp. 419-425, 2001.

[18] D. Gouwanda, N. Senanayake, M. Marasinghe et al., "Real time force sensing mat for human gait analysis," International Journal of Mechanical and Mechatronics Engineering, vol. 2, no. 3, pp. 349-354, 2008.

[19] B. Van Den Broeck, L. Vuegen, H. Van Hamme, M. Moonen, P. Karsmakers, and B. Vanrumste, "Footstep localization based on in-home microphone-array signals," in Proceedings of the 12th Biennial European Conference of the Association for the Advancement of Assistive Technology in Europe (AAATE 2013), vol. 33, p. 90, IOS Press, Amsterdam, Netherlands, September 2013.

[20] A. Helal, D. J. Cook, and M. Schmalz, "Smart home-based health platform for behavioral monitoring and alteration of diabetes patients," Journal of Diabetes Science and Technology, vol. 3, no. 1, pp. 141-148, 2009.

[21] J. Marsh, "House calls," in Rochester Review, vol. 64, no. 3, pp. 22-26, University of Rochester, Rochester, NY, USA, 2002, https://www.rochester.edu/pr/Review/V64N3/feature2. html.

[22] Georgia Tech, Aware Home Research Initiative, Georgia Institute of Technology, Atlanta, Georgia, 2017, http://www. awarehome.gatech.edu.

[23] N. Zouba, F. Bremond, and M. Thonnat, "An activity monitoring system for real elderly at home: validation study," in Proceedings of the 2010 Seventh IEEE International Conference on Advanced Video and Signal Based Surveillance (AVSS), pp. 278-285, IEEE, Boston, MA, USA, September 2010.

[24] A. Wood, J. Stankovic, G. Virone et al., "Context-aware wireless sensor networks for assisted living and residential monitoring," IEEE Network, vol. 22, no. 4, pp. 26-33, 2008.

[25] J. Brooke, "SUS-A quick and dirty usability scale," Usability Evaluation in Industry, vol. 189, no. 194, pp. 4-7, 1996.

[26] B. Laugwitz, T. Held, and M. Schrepp, "Construction and evaluation of a user experience questionnaire," in Symposium of the Austrian HCI and Usability Engineering Group, pp. 63-76, Springer, Graz, Austria, November 2008.

[27] A. Bangor, P. Kortum, and J. Miller, "Determining what individual SUS scores mean: adding an adjective rating scale," Journal of Usability Studies, vol. 4, no. 3, pp. 114-123, 2009. 

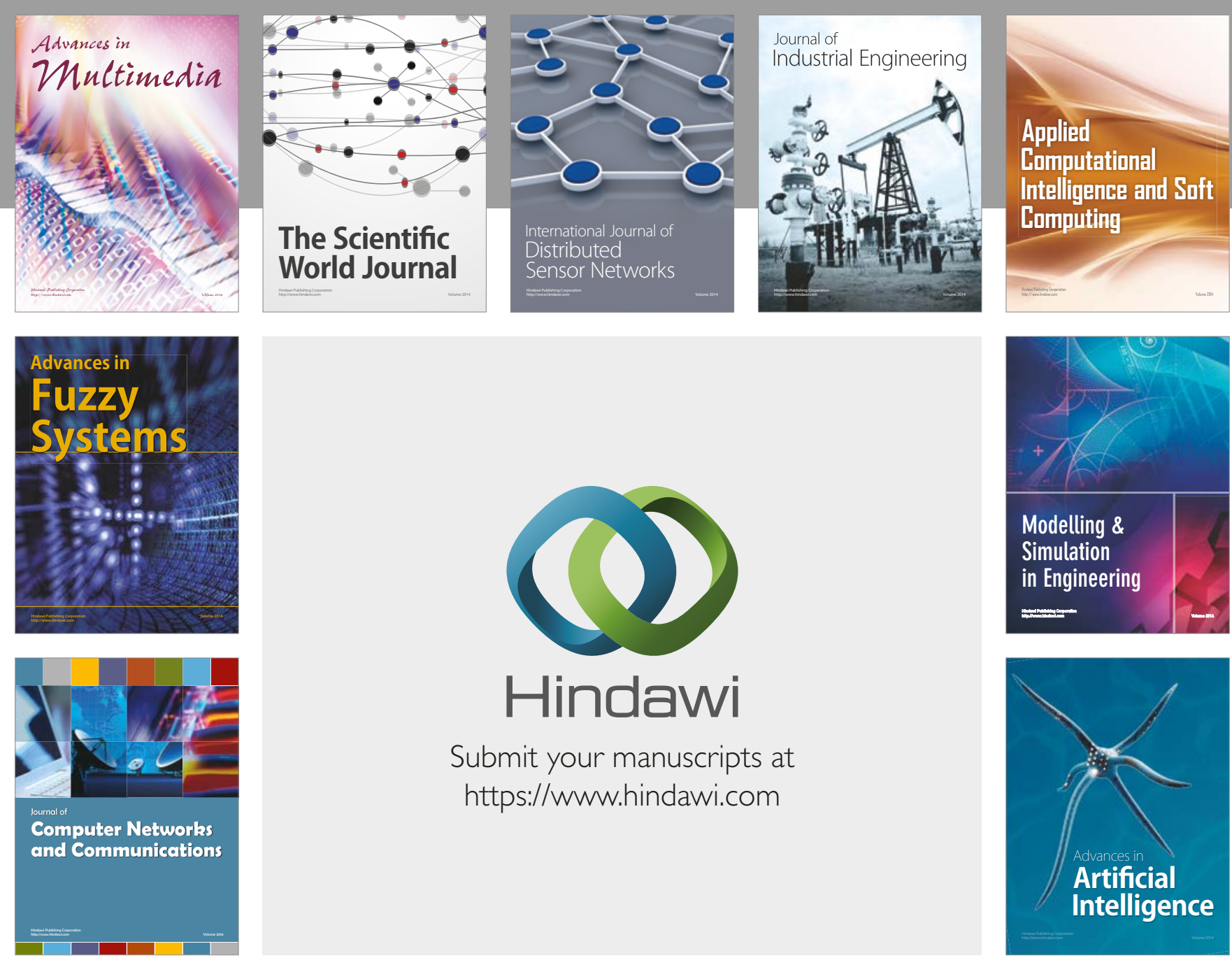

\section{Hindawi}

Submit your manuscripts at

https://www.hindawi.com
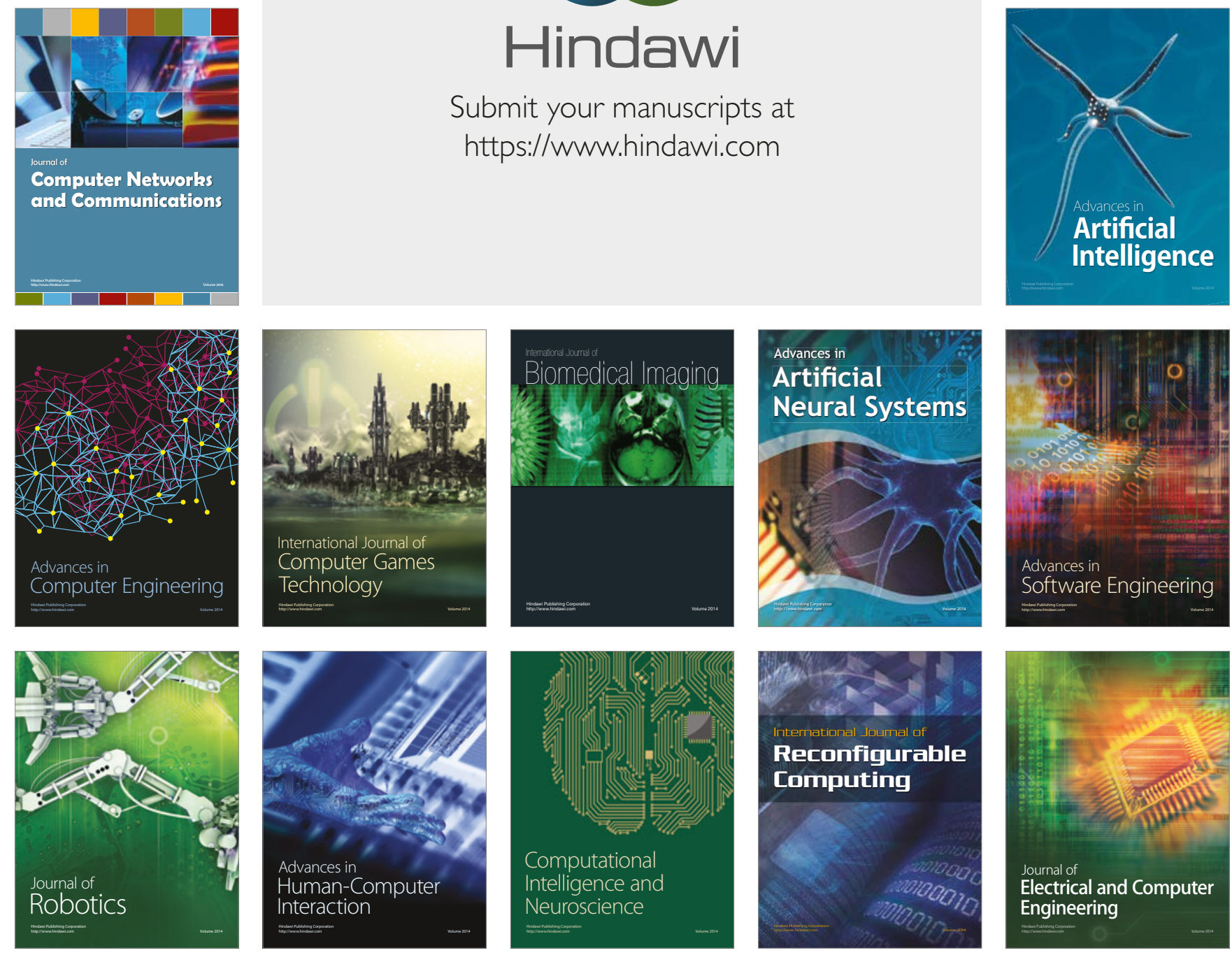